\title{
Prevalencia del Trastorno por Estrés Postraumático por la Guerra, en Niños de Cundinamarca, Colombia
}

\author{
Isabel Pérez-Olmos ${ }^{1}$, Patricia E. Fernández-Piñeres ${ }^{2}$ y Sonia Rodado-Fuentes ${ }^{3}$ \\ ${ }^{1}$ Médica. Especialista en Psiquiatría, Especialista en Psicoanálisis, Especialista en \\ Epidemiología. Facultad de Medicina, Universidad del Rosario, Bogotá, Colombia. \\ E-mail: iperez@urosario.edu.co \\ ${ }^{2}$ Médica. Especialista en Psiquiatría. Gerente Salud Pública. Hospital Nazareth. Bogotá, \\ Colombia. E-mail: patiferna@yahoo.com \\ ${ }^{3}$ Médica. Especialista en Psiquiatría. E.S.E. Centro de Atención y Rehabilitación Integral en \\ Salud. C.A.R.I. Barranquilla. E-mail: soniarodado@hotmail.com
}

Recibido 14 Junio 2005/Enviado para Modificación 23 Septiembre 2005/Aceptado 3 Octubre 2005

\section{RESUMEN}

Objetivos Determinar en tres municipios de Cundinamarca la prevalencia del Trastorno por Estrés Postraumático (TEPT) infantil y factores asociados, según tipo de exposición a la guerra.

Métodos Estudio de corte transversal. Muestra representativa de 493 escolares de 5 a 14 años de edad. 167 niños en La Palma, con exposición crónica; 164 en Arbeláez, con un hostigamiento armado reciente y 162 en Sopó, sin exposición. Por medio de entrevistas psiquiátricas semi-estructuradas y la Escala para Trastorno de Estrés Postraumático Administrada por el Clínico se determinó la prevalencia de TEPT y factores de vulnerabilidad, la razón de desventaja $(\mathrm{RD})$ y Ji-cuadrada. Se hizo análisis multivariado con regresión logística. Los niños afectados requirieron intervención de salud mental.

Resultados Prevalencia de TEPT bélico: La Palma 16,8 \%, Arbeláez 23,2 $\%$, y Sopó 1,2 \%. Al comparar los municipios expuestos con Sopó: RD 19,9 (IC 4,7, 119,2), Ji-cuadrada 30,4 P=0,000. La regresión logística identificó que la proximidad geográfica y la alteración emocional intensa con el evento estresante incrementaron el TEPT. Los factores de vulnerabilidad predominaron en los municipios expuestos a la guerra. Los indicadores de pobreza, baja escolaridad paterna y maltrato infantil predominaron en La Palma. En Arbeláez predominó el déficit de atención y los trastornos psicosomáticos.

Conclusiones La Guerra afecta la salud mental infantil. Los niños de los municipios expuestos tuvieron 19 veces mayor probabilidad de sufrir TEPT bélico que los no expuestos. Intervenir tempranamente es prioridad en salud pública. Los resultados son útiles para países con conflicto bélico o terrorismo. 
Palabras Clave: Trastornos de estrés postraumático, psiquiatría infantil, estudios epidemiológicos, guerra, psicológía, modelos logísticos, Colombia (fuente: DeCS, BIREME).

\section{ABSTRACT \\ The prevalence of war-related post-traumatic stress disorder in chil- dren from Cundinamarca, Colombia}

Objective Determining the prevalence of post-traumatic stress disorder (PTSD) related to the type of war exposure and associated factors in schoolaged children from three Colombian towns.

Methods Cross-sectional epidemiological study. Representative randomised sample of 493 children aged 5-14. The children were evaluated during 2002 using semi-structured psychiatric interviews and the clinician administered PTSD scale. 167 children were evaluated in La Palma who had been chronically exposed to war, 164 in Arbeláez who had had recent war-exposure and 162 in Sopó who had not been exposed to war. War-related PTSD prevalence was calculated in each municipality. Odds ratio (OR) and chi-square were used for evaluating the association between exposure to war and PTSD and the related risk. Multivariate analysis used the logistic regression model. The affected children required specialised mental health counselling. Results The prevalence of PTSD resulting from war was $16,8 \%$ in La Palma, 23,2 \% in Arbeláez and 1,2 \% in Sopó. A 19,9 OR (Cl 4.7, 119.2), 30,5 Chi-square and $p=0.000$ revealed war-related PTSD association and risk for children when comparing the exposed towns to Sopó. The logistic regression showed that geographical closeness to war zone and intense emotional reaction to war increased the probability of war-related PTSD. Vulnerability factors were predominant in war-exposed towns. Poverty, parents' low educational level and child abuse predominated in La Palma. Attention-deficit and psychosomatic disorders were more prevalent in $\mathrm{Ar}$ beláez.

Conclusions War affects children's mental health; the children from the exposed towns had 19 times greater probability of war-related PTSD than those from a non-exposed town. Early therapeutic intervention is a public health priority. The results are useful for countries suffering from war, internal conflict and/or terrorism.

Key Words: Stress disorder, post-traumatic, child psychiatry, epidemiological study, war, psychological test, logistic model, Colombia (source: MeSH, $N L M)$.

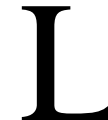

a Guerra es una experiencia colectiva que destruye el mundo social de la población afectada. En Colombia ha existido el conflicto armado interno en el último medio siglo. En las dos últimas décadas éste no sólo aumentó, sino que se extendió en el país (1). El riesgo de morir 
o enfermar como víctima de la guerra aumentó así como el desplazamiento forzado y la pobreza de las comunidades afectadas (1).

Las reacciones por estrés incluyen ansiedad, aturdimiento, recuerdos del trauma y alteración de la atención. Un trastorno por estrés se diferencia de la reacción normal por su mayor severidad, la diversidad de síntomas y la alteración del funcionamiento social (2). El trastorno por estrés agudo ocurre en el primer mes (2-4). En los siguientes seis meses pueden ocurrir síndromes depresivos, ansiosos, psicosomáticos y el trastorno por estrés postraumático (TEPT) (2-4). Los niños pueden tener trastornos de conducta, de aprendizaje, reacciones violentas o intentos de suicidio (5). Algunos estudios han identificado que las mujeres y las personas más expuestas al trauma o con psicopatología previa tienen mayor riesgo de TEPT (6). Otros señalan al bajo estatus educativo y socioeconómico como riesgo para TEPT (6). La resiliencia protege de la adversidad, sin embargo su conocimiento científico es aún escaso.

La prevalencia de TEPT alguna vez en la vida en la población mundial es de 1 a $4 \%$ (7). Varía según factores como el tipo de evento estresante, la edad o el sexo (8-9). Savin y cols., 1996 (10), reportaron en Tailandia una prevalencia de TEPT de 71,7 \% en niños camboyanos del campamento de refugiados, mientras Sack y cols. (11), reportaron una prevalencia de TEPT de $50 \%$ en niños de igual procedencia. En Colombia hay pocos estudios de prevalencia de TEPT; el $2^{\circ}$ Estudio Nacional de Salud mental y consumo de sustancias psicoactivas de 1997 (12) mostró una prevalencia de TEPT, alguna vez en la vida, en jóvenes de 4,3\%. Si el TEPT no recibe tratamiento adecuado puede evolucionar a deterioro permanente del funcionamiento individual y social (13-14).

Si el trauma es repetido en la infancia puede deformar la estructuración de la personalidad (15). Diferentes factores influyen en la vulnerabilidad y la resiliencia de los niños frente al estrés (16-17). Pynoss y cols. (18), evaluaron niños de una escuela atacada por un francotirador y encontraron prevalencias de TEPT en quienes acudieron al colegio ese día de 94,3\%, 45 \% en los que no asistieron y $89 \%$ en quienes estaban dentro del edificio.

Herman (14) describe que la cercanía de los padres tranquiliza al niño estresado y le da seguridad. Si el entorno familiar tiene pobre resiliencia es poco probable que esta seguridad se logre. El niño debe compensar las fallas de los cuidadores con un sistema psicológico de defensa inmaduro (19). La respuesta inicial del niño por un gran estrés es un estado de alerta con pobre concentración, y puede confundirse con un trastorno por déficit de atención 
con o sin hiperactividad (TDA/H) (14). Mc Leer y cols. (20) encontraron una prevalencia de TEPT del $40 \%$ en niños abusados sexualmente y un tercio de ellos tuvo criterios para TDA/H. La sensación de inseguridad restringe las exploraciones y la autoestima de los niños. El infante afectado disminuye su creatividad en la solución de problemas e incluso puede tener conductas impulsivas o di sociales (14).

La identificación temprana de individuos con mayor riesgo de TEPT y la intervención oportuna es una tarea prioritaria en la salud mental pública (2123). Los estudios de prevalencia ofrecen un diagnóstico rápido y económico. Este estudio de prevalencia de TEPT por exposición al conflicto armado en una muestra poblacional representativa de niños brinda información útil para la intervención en salud mental. Se realizó en el 2002, con escolares de tres municipios de Cundinamarca con exposiciones diferentes al estrés bélico.

\section{MATERIALES Y MÉTODOS}

Es un estudio de corte transversal, la variable dependiente fue TEPT por exposición bélica y las independientes, el municipio, tipo de exposición a la guerra y otros factores de vulnerabilidad y protección.

\section{Participantes}

Se seleccionaron tres municipios con diferente exposición a la guerra, accesibilidad geográfica y colaboración de las autoridades locales: La Palma, con exposición crónica, frecuentes víctimas humanas y daños físicos. Arbeláez con una exposición 6 meses atrás que dejó dos muertos y la destrucción de edificaciones públicas y privadas. Sopó, sin exposición. La Jefatura de emergencias y desastres de Cundinamarca facilitó el transporte amparado en el Programa Misión Médica del Ministerio de Salud, enmarcado en el Derecho Internacional Humanitario (24).

Marco y tamaño muestral

Se incluyeron niñas/os de 5 a 14 años, inscritos en el sistema educativo público y privado. Se excluyeron niños no residentes al menos 6 meses en el municipio. La selección fue aleatoria y proporcional al número de niños registrados en cada institución educativa para lograr una muestra representativa de la población infantil escolar de cada municipio.

Calculo del tamaño muestral con Epi-info 6, confianza $95 \%$, poder $80 \%$, prevalencia de TEPT en población general, $5 \%$, y $15 \%$ en expuestos, rela- 
ción 1:1 entre expuestos y no expuestos, razón de desventaja (RD) 3,4. Muestra obtenida, 159 niños por municipio, total 477 niños. Adición $3 \%$ para suplir pérdidas.

Instrumentos y medidas

Tres residentes de psiquiatría de III año de la Facultad de Medicina de la Universidad del Rosario realizaron entrevistas psiquiátricas semi-estructuradas a los niños acompañados de sus cuidadores y aplicaron la versión española de la Escala para el Trastorno de Estrés Postraumático Administrada por el Clínico, (Clinician Administered PTSD Scale, CAPS), obtenida del libro con su validación (25). El CAPS se basa en el DSM IV, evalúa los criterios diagnósticos de TEPT $(2,25)$. Este instrumento era el único validado para hispanos en el momento del estudio (25). No se disponía de la versión española del CAPS de niños y adolescentes. La escala funcionó adecuadamente, quizá por el uso de los criterios especificados para TEPT infantil del DSM IV (2).

El formato de recolección de información de la entrevista psiquiátrica incluyó antecedentes de enfermedad, factores sociodemográficos (edad y género, escolaridad de los padres, estrato socioeconómico), factores de vulnerabilidad y protección, ubicación geográfica y alteración emocional intensa del niño y familiares durante la exposición y criterios diagnósticos de trastornos psiquiátricos infantiles (2). Se indagó por existencia de maltrato infantil, desplazamiento forzado, muerte de algún familiar, madresolterismo y resiliencia familiar y del entorno (26). El rendimiento escolar, las emociones predominantes y los rasgos de personalidad del niño se obtuvieron a partir de la opinión de los padres.

Análisis de datos

Con la información recolectada se crearon bases de datos en Excel (Microsoft). El análisis estadístico se hizo con SPSS 10.0 para Windows y EpiInfo 6. Se calculó la prevalencia de TEPT bélico infantil y las frecuencias de cada variable. A las variables cuantitativas se les calcularon promedios y desviaciones estándar y a las variables cualitativas sus proporciones.

Se exploraron asociaciones entre las variables. Se calculó la ji-cuadrada y la razón de desventaja (RD) para el riesgo de TEPT por exposición bélica. El nivel de significación estadística fue $5 \%$. Se hizo análisis multivariado con la regresión logística de Hosmer y Lemeshow (27). El análisis estadístico 
con la información codificada permitió el enmascaramiento de la identidad de los menores evaluados.

\section{Consideraciones éticas}

El estudio estuvo enmarcado en las normas éticas y jurídicas vigentes en Colombia, acordes con la Declaración de Helsinki actualizada en Escocia, Octubre, 2000. Hubo aprobación escrita de los alcaldes y comités de ética de los hospitales municipales, consentimiento informado de los padres y asentimiento de los niños. Los niños con TEPT fueron remitidos a atención clínica por equipo de psiquiatría y salud mental infantil. Los resultados de esa intervención no se presentan en este artículo. La financiación fue provista por los investigadores y la Facultad de Medicina de la Universidad del Rosario. Los autores declaran no tener conflictos de intereses.

\section{RESULTADOS}

Se evaluaron 493 niños en los tres municipios. 164 en Arbeláez (tasa de respuesta $100 \%$ ), edad promedio 11,6 años. 167 en La Palma, (tasa de respuesta $100 \%$ ), edad promedio 9,9 años. 162 en Sopó, (tasa de respuesta 99 $\%)$, edad promedio 10,5 años, Tabla 1. Las frecuencias de las variables sociodemográficas sexo, edad, escolaridad y estrato socioeconómico de los padres se encuentran en la Tabla 1.

Tabla 1. Descripción socio-demográfica de los niños evaluados en cada municipio

\begin{tabular}{|c|c|c|c|c|c|c|}
\hline Variables & Categorías & $\begin{array}{c}\text { Arbeláez } \\
\mathrm{N}=164 \\
(\%)\end{array}$ & $\begin{array}{c}\text { La Palma } \\
\mathrm{N}=167 \\
(\%)\end{array}$ & $\begin{array}{c}\text { Sopó } \\
\mathrm{N}=162 \\
(\%)\end{array}$ & $\begin{array}{c}\text { Total } \\
\mathrm{N}=493 \\
(\%)\end{array}$ & $\begin{array}{l}\text { Valor } \\
\text { p }\end{array}$ \\
\hline \multirow{2}{*}{ Género } & $\mathrm{F}$ & $78(47,6)$ & $77(46,1)$ & $86(53,1)$ & $241(48,9)$ & \multirow{2}{*}{0,412} \\
\hline & $M$ & $86(52,4)$ & $90(53,9)$ & $76(46,9)$ & $252(51,1)$ & \\
\hline Edad & $\begin{array}{l}\text { Promedio } \\
\text { en años } \\
\text { (desviación } \\
\text { estándar) }\end{array}$ & $11,6(2,8)$ & $9,9(2,9)$ & $\begin{array}{l}10,5 \\
(2,4)\end{array}$ & $10,6(2,8)$ & 0,01 \\
\hline \multirow{4}{*}{$\begin{array}{l}\text { Nivel de } \\
\text { Escolaridad de } \\
\text { los Padres }\end{array}$} & Analfabeta & $1(0,6)$ & $11(6,6)$ & $0(0)$ & $12(2,4)$ & \multirow{4}{*}{0,000} \\
\hline & Primaria & $98(59,8)$ & $111(66,5)$ & $73(45,1)$ & $282(57,2)$ & \\
\hline & Secundaria & $53(32,3)$ & $45(26,9)$ & $78(48,1)$ & $176(35,7)$ & \\
\hline & Universitaria & $12(7,3)$ & $0(0)$ & $11(6,8)$ & $23(4,7)$ & \\
\hline \multirow{5}{*}{$\begin{array}{l}\text { Estrato } \\
\text { Socioeconómico }\end{array}$} & 1 & $53(32,3)$ & $55(32,9)$ & $8(4,9)$ & $116(23,5)$ & \multirow{5}{*}{0,000} \\
\hline & 2 & $81(49,4)$ & $100(59,9)$ & $86(53,1)$ & $267(54,2)$ & \\
\hline & 3 & $20(12,2)$ & $9(5,4)$ & $56(34,6)$ & $85(17,2)$ & \\
\hline & 4 & $8(4,9)$ & $3(1,8)$ & $12(7,4)$ & $23(4,7)$ & \\
\hline & 5 & $2(1,2)$ & $0(0)$ & $0(0)$ & $2(0,4)$ & \\
\hline
\end{tabular}

Arbeláez: única exposición bélica reciente. La Palma: exposición bélica crónica; Sopó: municipio sin exposición bélica directa a la fecha del estudio. 
No hubo diferencia significativa en la distribución por sexo en los tres municipios, pero sí en las otras variables sociodemográficas. Los estratos socioeconómicos bajos y el analfabetismo de los padres fue mayor en La Palma (Tabla 1). El 54,2 \% de los niños estuvo expuesto al menos a un evento estresante de origen bélico. La prevalencia de TEPT (agudo y crónico) en los niños del municipio de Arbeláez fue 23,2 \%, en La Palma 16,8 \% y en Sopó, 1,2 \% (Figura 1).

Al comparar los casos de TEPT bélico de los municipios expuestos al conflicto armado, Arbeláez y La Palma, con Sopó, se encontró un riesgo significativo de TEPT bélico RD: 19,9, IC 4,7, 119,2; Ji-cuadrada (corrección de Yates): 30,4 p= 0,000. Al comparar Arbeláez, con Sopó, se encontró un riesgo significativo de TEPT bélico, RD: 24,3, IC 5,5, 147,4; Ji-cuadrada (corrección de Yates): 34,4, p=0,000. Al comparar La Palma con Sopó se encontró un riesgo significativo de TEPT bélico, RD: 16,1, IC 3,6, 99,6; Jicuadrada (corrección de Yates): 22,1, p= 0,0000026.

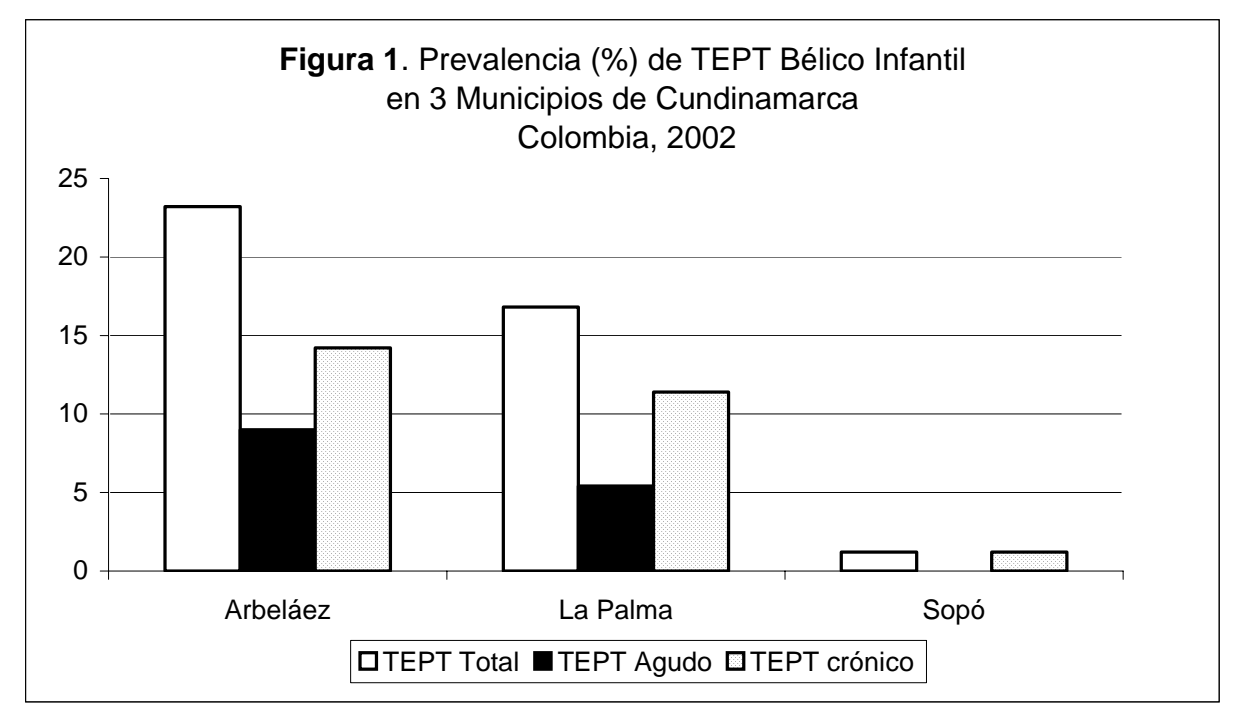

El TEPT bélico fue más frecuente, 32,4 \%, cuando el afectado se encontraba en la calle durante el evento traumático en comparación a si estaba en la casa o la escuela 20,4\%, o fuera del municipio 9,5 \% p=0,000. Las frecuencias de otros factores de vulnerabilidad y protección se muestran en la Tabla 2. 
También se encontró que el 33,7 \% de los niños con TEPT bélico había presentado una reacción emocional más intensa que lo habitual durante el evento traumático, Ji-cuadrada (corrección de yates): 79,3 p=0,000.

En el 35,7 \% de los casos de TEPT bélico tanto el niño como un adulto cercano tuvieron una alteración emocional más intensa, Ji-cuadrada (corrección de Yates): 61,5; RD: 3,0 IC: 2,4, 3,9; p=0,000. También tuvo significación estadística la asociación entre TEPT bélico y la capacidad deficiente de resiliencia familiar RD: 1,4, IC: 1,1, 1,8; Ji-cuadrada, corrección de Yates, 5,9; $\mathrm{p}=0,015$; y el predominio de características infantiles como aislamiento, inquietud o ansiedad, Ji-cuadrada, corrección de Yates: 5,9; RD: 2,2 IC: 1,1, 4,$0 ; p=0,015$.

Tabla 2. Frecuencia de algunos factores de vulnerabilidad y

\begin{tabular}{|c|c|c|c|c|c|}
\hline Factores asociados & $\begin{array}{c}\text { Arbeláez } \\
\mathrm{N}=164 \\
(\%)\end{array}$ & $\begin{array}{l}\text { La Palma } \\
\mathrm{N}=167 \\
(\%)\end{array}$ & $\begin{array}{c}\text { Sopó } \\
\mathrm{N}=162 \\
(\%)\end{array}$ & $\begin{array}{c}\text { Total } \\
\mathrm{N}=493 \\
(\%)\end{array}$ & $\begin{array}{l}\text { Valor } \\
p\end{array}$ \\
\hline Desplazamiento forzado & $12(7,3)$ & $34(20,4)$ & $4(2,5)$ & $50(10,1)$ & 0,000 \\
\hline Muerte de familiar & $21(12,8)$ & $55(32,9)$ & $26(16,0)$ & $102(20,7)$ & 0,000 \\
\hline Madresolterismo & $36(22,0)$ & $40(24,0)$ & $16(9,9)$ & $92(18,7)$ & 0,006 \\
\hline $\begin{array}{l}\text { Bajo ingreso económico } \\
\text { (BIE) }\end{array}$ & $90(54,9)$ & $121(72,5)$ & $50(30,9)$ & $261(52,9)$ & 0,000 \\
\hline $\begin{array}{c}\text { Familia Multi- } \\
\text { problemática (FM) }\end{array}$ & $97(59,1)$ & $121(72,5)$ & $50(30,9)$ & $268(54,4)$ & 0,000 \\
\hline $\begin{array}{l}\text { Rendimiento escolar } \\
\text { adecuado }\end{array}$ & $101(61,6)$ & $93(55,7)$ & $113(69,8)$ & $307(62,3)$ & 0,036 \\
\hline $\begin{array}{l}\text { Deficiente adaptabilidad } \\
\text { individual }\end{array}$ & $27(16,5)$ & $40(24,0)$ & $11(6,8)$ & $78(15,8)$ & 0,000 \\
\hline $\begin{array}{l}\text { Deficiente adaptabilidad } \\
\text { familiar }\end{array}$ & $77(47,0)$ & $90(53,9)$ & $12(7,4)$ & $179(36,3)$ & 0,000 \\
\hline $\begin{array}{l}\text { Deficiente adaptabilidad } \\
\text { del entorno }\end{array}$ & $4(2,4)$ & $141(84,4)$ & $31(19,1)$ & $176(35,7)$ & 0,000 \\
\hline $\begin{array}{l}\text { Deficiente resiliencia } \\
\text { (individual, familiar y } \\
\text { comunitaria) }\end{array}$ & $10(6,1)$ & $121(72,5)$ & $4(2,5)$ & $135(27,4)$ & 0,000 \\
\hline Aislamiento infantil & $30(18,3)$ & $17(10,2)$ & $15(9,3)$ & $62(12,6)$ & 0,025 \\
\hline
\end{tabular}

Nota: BIE: menos de $\$ 305000$ pesos colombianos de ingreso mensual. FM: existencia de $\mathrm{BIE}$, madresolterismo o padres ancianos y más de 4 hijos.

Así mismo, otros factores estresantes como desplazamiento forzado, la muerte de un familiar, el madresolterismo, el bajo ingreso económico, la familia multiproblemática, la deficiente adaptabilidad individual, familiar y del entorno tuvieron frecuencias dobles o triples en La Palma comparados con Arbeláez y Sopó (Tabla 2). Los niños sin TEPT tuvieron mayor frecuencia de rendimiento escolar adecuado según la opinión de los padres. 
El maltrato físico y psicológico, trastornos de aprendizaje y comportamiento predominaron en La Palma seguidos de Arbeláez. El déficit de atención y los trastornos psicosomáticos fueron más frecuentes en Arbeláez. En Sopó hubo algunos casos de abuso sexual (Tabla 3).

Tabla 3. Frecuencia de criterios diagnósticos psiquiátricos infantiles según municipio

\begin{tabular}{|c|c|c|c|c|c|}
\hline $\begin{array}{c}\text { Critérios } \\
\text { diagnósticos } \\
\text { psiquiátricos } \\
\text { infantiles } \\
\text { (DSM IV) }\end{array}$ & $\begin{array}{c}\text { Arbeláez } \\
\mathrm{N}=164 \\
(\%)\end{array}$ & $\begin{array}{l}\text { La Palma } \\
\mathrm{N}=167 \\
(\%)\end{array}$ & $\begin{array}{c}\text { Sopó } \\
N=162 \\
(\%)\end{array}$ & $\begin{array}{c}\text { Total } \\
\mathrm{N}=493 \\
(\%)\end{array}$ & $\begin{array}{c}\text { Valor } \\
\text { P }\end{array}$ \\
\hline $\begin{array}{l}\text { Trastorno de } \\
\text { aprendizaje }\end{array}$ & $19(11,6)$ & $31(18,6)$ & $11(6,8)$ & $61(12,4)$ & 0,005 \\
\hline Maltrato físico & $31(18,9)$ & $56(33,5)$ & $27(16,7)$ & $114(23,1)$ & 0,002 \\
\hline $\begin{array}{c}\text { Maltrato } \\
\text { psicológico }\end{array}$ & $14(8,5)$ & $17(10,2)$ & $15(9,3)$ & $46(9,3)$ & 0,688 \\
\hline Maltrato sexual & 0 & 0 & $3(1,9)$ & $3(0,6)$ & 0,086 \\
\hline $\begin{array}{l}\text { Trastorno de } \\
\text { lenguaje }\end{array}$ & $3(1,8)$ & $3(1,8)$ & $1(0,6)$ & $7(1,4)$ & 0,574 \\
\hline $\begin{array}{l}\text { Trastorno de } \\
\text { conducta }\end{array}$ & $29(17,7)$ & $27(16,2)$ & $12(7,4)$ & $68(13,8)$ & 0,015 \\
\hline $\begin{array}{l}\text { Trastorno } \\
\text { depresivo }\end{array}$ & $31(18,9)$ & $38(22,8)$ & $27(16,7)$ & $96(19,5)$ & 0,369 \\
\hline $\begin{array}{l}\text { Déficit de } \\
\text { atención con/sin } \\
\text { hiperactividad }\end{array}$ & $34(20,7)$ & $31(18,6)$ & $28(17,3)$ & $93(18,9)$ & 0,723 \\
\hline $\begin{array}{c}\text { Trastorno } \\
\text { psicosomático }\end{array}$ & $49(29,9)$ & $48(28,7)$ & $50(30,9)$ & $147(29,8)$ & 0,377 \\
\hline
\end{tabular}

El modelo de regresión logística mostró un ajuste significativo (Prueba de Hosmer y Lemeshow, $p=0,9$ ). El TEPT fue más probable si el niño estuvo en la calle durante el evento estresante (RD 3,3) y si se alteró emocionalmente más allá de lo esperable con el estrés (RD 3,3).

\section{DISCUSION}

La prevalencia de TEPT en Arbeláez y La Palma, expuestos a la guerra, fue superior al 1-4 \% de la población mundial (7) y al 4,3 \% de población juvenil colombiana (12). Sin embargo, estuvo dentro del rango presentado en estudios internacionales (7,10-11).

Los niños de Arbeláez y La Palma tuvieron 19 veces mayor riesgo de TEPT bélico que los de Sopó. La exposición a la guerra es un factor de riesgo para TEPT infantil de causa bélica. Algunos intervalos de confianza 
de la RD fueron amplios. Esto puede ocurrir cuando la muestra es pequeña o cuando hay gran variabilidad en las características que definen el caso, así los criterios diagnósticos del DSM IV para TEPT incluyen 18 ítems posibles en los 4 criterios indispensables A, B, C y D (2).

Consistente con lo señalado por algunos autores $(14,20)$ acerca del estrés reciente y la superposición de criterios de TEPT y TDA/H, hubo mayor prevalencia de TDA/H en niños de Arbeláez y trastornos psicosomáticos que se asocian también con ansiedad y estrés.

El rasgo de conducta, según la percepción de los padres, más frecuente en La Palma y Arbeláez fue el aislamiento infantil, esta característica está incluida en el diagnóstico de TEPT Complejo propuesto por Herman (14).

El promedio de edad de los niños en La Palma fue significativamente menor, puede deberse a que el conflicto armado crónico provoca mayor deserción escolar y vinculación de niños a los grupos armados ilegales y también por su migración protectora. Al respecto, el representante del Banco Mundial en Colombia (28) informó que la deserción escolar infantil es de $30 \%$ en zona rural y $18 \%$ urbana. Así, los menores evaluados representarían entre el $70 \%$ y $82 \%$ de los niños de estos municipios (28). Hubo mayor riesgo de TEPT, si el niño o alguno de sus familiares habían tenido alteración emocional intensa con el evento estresante (RD: 20,6, $\mathrm{p}<0,05)$, este hallazgo es consistente con lo planteado por González (29). Algunos estudios plantean que trastornos psiquiátricos previos como la depresión y la ansiedad conllevan mayor riesgo para TEPT $(6,16)$, estos trastornos también fueron más frecuentes en los municipios expuestos; sin embargo, una limitación del estudio de corte transversal es que no se puede establecer si esta psicopatología antecedía o no a la exposición bélica.

La proximidad geográfica y la alteración emocional intensa durante el trauma aumentaron el riesgo de TEPT, estas dos variables indicarían la amenaza sobre la integridad personal y la indefensión experimentadas por la víctima $(6,18,19,22,29)$. También ciertas variables sociodemográficas y económicas se asocian en forma significativa con TEPT e indican deterioro social (16). Hubo asociaciones significativas de TEPT y deficiencia de la resiliencia familiar, 89,6 \% de los casos de TEPT en La Palma y Arbeláez tenían esta condición. El riesgo de TEPT en estas familias fue casi el doble que en los otros casos; esto ilustra el importante rol de la red sociofamiliar.

Otros aspectos socio familiares fueron la mayor prevalencia en La Palma del maltrato físico, trastornos de aprendizaje y del comportamiento; al res- 
pecto cabe anotar que Garbarino y Eckenrode (30) describen cómo los niños afectados crónicamente por estrés crean una imagen negativa de si mismos que los lleva a habituarse a recibir castigo y retroalimentar el maltrato de sus cuidadores. La Palma tuvo mayor prevalencia de deficiencia en la adaptabilidad individual, familiar y comunitaria que los otros municipios. Estas características podrían ser efectos del estrés crónico por la guerra y signos del deterioro de la salud mental y calidad de vida comunitarios. Sin embargo, dilucidar estas dinámicas sociales requiere de estudios adicionales.

Algunos casos de estrés agudo tienen remisión espontánea, otros persisten por largos periodos. La temprana identificación e intervención de individuos con riesgo incrementado para TEPT debe considerarse una prioridad de la salud pública (21-23). Es necesario identificar los predictores de TEPT para programar intervenciones tempranas efectivas. No se debería esperar a que los hechos bélicos de nuestro país finalicen para implementar estrategias de prevención e intervención temprana de los niños y familias en comunidades afectadas. Los resultados presentados no pueden generalizarse al resto de niños colombianos •

Agradecimientos. Dr. Hernández y cols. Secretaría de Salud, Desarrollo Social y Educación, Cundinamarca. Cruz Roja Bogotá y Cundinamarca. Dr. German Casas y cols. Salud Mental, Instituto Roosevelt. Alcaldías, Escuelas, Colegios, Hospitales, municipios Arbeláez, La Palma y Sopó. Dr. A. Julio, investigador residente III Psiquiatría, Dra. Olga Albornoz, Dpto. Psiquiatría. Milciades Ibañez y Maria Nelcy Rodríguez, asesores estadísticos. Drs. Jaime Enrique Ruiz y Catalina Latorre, Grupos de Investigación Clínica y Salud Pública, Facultad de Medicina, Universidad del Rosario.

\section{REFERENCIAS}

1. PNUD. Informe Nacional de Desarrollo Humano para Colombia. El Conflicto, callejón con salida. Bogotá: Editorial El Malpensante; 2003.

2. American Psychiatric Association. Diagnostic and Statistical Manual of Mental Disorders, 4th Edition (DSM-IV). Washington, DC: American Psychiatric Association; 1995.

3.Ursano R. Post-Traumatic Stress Disorder. N Engl J Med. 2002; 346(2):130-135.

4.Van der Kold BA, McFarlane AC. The Black Hole of Trauma. In: Van der Kolk BA, McFarlane AC, Weisaeth L, editors. Traumatic Stress: The effects of overwhelming experience on mind, body and society. New York: The Guilford Press; 1996. p. 3-23.

5. Cardona D, Londoño G. Experiencias de Atención en Salud Mental después del 
terremoto del 25 de Enero de 1999 en Pereira. [Internet] Congreso Virtual de Psiquiatría 1 febrero - 7 Marzo Interpsiquis 2001. . Disponible en: http://www.psiquiatria.com Consultado Octubre 2001.

6. North CS, Westerhaus ET. Applications from previous disaster research to guide mental health interventions after the september 11 attacks. In: Ursano RJ, Fullerton CS, Norwood AE, editors. Terrorism and Disaster Individual and Community Mental Health Interventions. 1a. ed. Nueva York: Cambridge University Press; 2003. p. 93-106.

7. Kessler RC, Sonnega A, Bromet E, Hughes M, Nelson CB. Postraumatic Stress disorder in the National Comorbidy Survey. Arch. Gen Psychiatry. 1995; 52: $1048-1060$.

8. Cohen J. Practice parameter for the assessment and treatment of children and adolescents with posttraumatic stress disorder. J Am Acad Child Adolesc Psychiatry. 1998; 37 (supl. 10): 4-26.

9. Helzer J, Robins L, McEvoy L. Posttraumatic stress disorder in the general population. N Engl J Med. 1987; 317: 1630-1634.

10. Savin D, Sack W, Clarke G. The Khmer project: III. A study of trauma from Thailand's site II refugee camp. J Am Acad Child Adolesc Psychiatric. 1996; 35:384-391.

11. Sack W, Him C, Dickason D. Twelve-year follow-up study of Khmer youths who sufered massive war trauma as children. J Am Child Adolesc Psychiatry. 1999; 38:1173-1179.

12. Ministerio de Salud. Segundo Estudio Nacional de Salud Mental y Consumo de Sustancias Psicoactivas. Colombia; 1997.

13. Bramsen I., Van der Ploeg H. Fifty year later: The long term psychological adjustment of ageing World War II survivor. Acta Psychiatrica Scandinava. 1999; 100:350-358.

14. Herman J. Trauma and recovery The aftermath of violence from domestic abuse to political terror. $2^{\circ}$ ed. New York: Basic Books; 1997.

15. McFarlane AC, Van der Kolk BA. Trauma and Its Challenge to Society. In: Van der Kolk BA, McFarlane AC, Weisaeth L, editors. Traumatic Stress: The Effects of Overwhelming Experience on Mind, Body, and Society. New York: The Guilford Press; 1996.

16. Echeburúa E, Corral P, Amor P. Perfiles diferenciales del Trastorno de estrés Postraumático en distintos tipos de víctimas. Revista Análisis y Modificación de la Conducta.1998; 24: 527-555.

17. Foa EB, Hearst-Ikeda D, Perry KJ. Evaluation of brief cognitive-behavioral program for the prevention chronic PTSD in recent assault victims. J Consult Clin Psychol. 1995; 63:948-955.

18. Pynoss R, Frederick C, Nader N. Life threat and postraumatic stress in schoolage children. Arch Gen Psychiatric. 1987; 44:1057-1063.

19. Yehuda R, McFarlane A. Biology of Postraumatic stress disorder. J Clin Psychiatry. 2000; 61 (Supl.7): 14-21.

20. McLeer SV, Deblinger E, Atkins MS y cols. Traumatic stress disorder in sexually abused children. J Am Acad Child Adolesc Psychiatry. 1988; 27:650-654 
21. Golier J, Yehuda R. Neuropsychological processes in post-traumatic stress disorder. Psychiatr. Clin. N. Am. 2002; 25: 295-315.

22. Orr SP, Metzger LJ, Pitman RK. Psychophysiology of post-traumatic stress disorder. Psychiatr. Clin. N. Am. 2002; 25: 271-293.

23. Rabois D, Batten SV, Keane TM. Implications of biological findings for psychological treatments of post-traumatic stress disorder. Psychiatr. Clin. N. Am. 2002; 25: 443-462.

24. Ministerio de Salud de Colombia Resolución No. 01020 de 2002 Protección a la Misión Médica. Bogotá (Colombia): 2002.

25. Bobes J, Bousoño M, Calcedo A, González MP. Trastorno de Estrés Postraumático. $1^{\text {a }}$ ed. Barcelona: Masson; 2000.

26. Linares JL. Identidad y Narrativa. Barcelona: Paidos; 1996.

27. Hosmer DW, Lemeshow S. Applied Logistic Regression. New York: John Wiley \& Sons; 1989.

28. Chueca A. La deserción no cede. Periódico El Tiempo. Sección: Educación. Publicación: Bogotá (Col. 1); 2003 Nov 9.

29. González ME, Medina JL, Pérez-Iñigo JL, Robles JI, Gómez-Trigo J. Conductas individuales y reacciones ante una situación de catástrofe. Madrid: Gomez Ulla; 2002.

30. Garbarino J, Eckenro de J.¿Por qué las familias abusan de sus hijos?. Buenos Aires: Ediciones Grancia; 1999. 